\title{
Das Prostatakarzinom des Hundes
}

\author{
Franziska Eberhardt, Ingmar Kiefer
}

Prostatatumoren beim Hund sind selten $[31,50]$ : In der Literatur wird die Prävalenz mit 0,2-0,6\% angegeben [8]. Im Vergleich dazu ist das Prostatakarzinom beim Mann mit etwa 26\% die häufigste Krebserkrankung in Deutschland [15]. Aufgrund der Vorsorgeuntersuchungen und Früherkennungsprogramme liegt das Sterberisiko jedoch lediglich bei $6 \%$ [15].

\section{Definition des Prostatakarzinoms}

In der Humanmedizin ist eine Prostataneoplasie histologisch zu etwa 94\% ein Adenokarzinom [2]. Daher ist in der Regel ein Adenokarzinom gemeint, wenn von einem Prostatakarzinom gesprochen wird. In der Veterinärmedizin hingegen werden verschiedene Tumoren unter dem Begriff „Prostatakarzinom“ zusammengefasst. Zu den häufigsten Prostatatumoren gehören:

- Adenokarzinome (am häufigsten)

$[13,18,28,31,50]$

- Übergangsepithelkarzinome

- Plattenepithelkarzinome

- undifferenzierte Sarkome $[9,44,61]$

Dabei scheinen die Übergangsepithelkarzinome wahrscheinlich aus dem urothelialen Epithel des intraprostatisch verlaufenden Urethraanteils zu entstehen und sich in die Prostata und die Blase auszubreiten [44]. Bei den Adenokarzinomen gibt es unterschiedliche Differenzierungsformen [44]. Histologisch kann zwischen urothelialen, glandulären, squamoiden und sarkomatösen Anteilen unterschieden werden $[13,33$, $44,46,55]$. Dagegen sind mesenchymale Tumoren der Prostata sehr selten [44]. Es gibt Einzelfallbeschreibungen von primären Leiomyosarkomen $[6,26]$, primär malignen Lymphomen $[4,9,55,61]$ und Hämangiosarkomen [14, 25].

\section{Vorkommen}

Das durchschnittliche Alter der erkrankten Hunde liegt bei etwa 10 Jahren $[8,13$, 31,50,51,52,55]. Dabei treten Prostatakarzinome bei kastrierten Rüden signifikant häufiger als bei unkastrierten Hunden auf $[8,10,31,51,55]$.

\section{konkret}

Das Risiko, an einem Prostatakarzinom zu erkranken, ist bei kastrierten

Hunden um das 3,86-fache erhöht [10].

Das Risiko, speziell an einem Adenokarzinom zu erkranken, ist bei kastrierten Rüde um das 2,12-fache erhöht [10]. Dabei scheinen Rasseprädispositionen eine Rolle zu spielen [10]. Unter anderem wird für folgende Rassen eine statistisch signifikantere Wahrscheinlichkeit angegeben, eine Prostataneoplasie zu entwickeln [10]:

- Dobermann

- Airedale Terrier

- Scottish Terrier

- Deutsch Kurzhaar

Andere Autoren beschreiben, dass für Rüden der Rasse Bouvier des Flandres das Risiko, an einem Prostatakarzinom zu erkranken, um das 8,4-fache erhöht ist [55].

\section{Pathogenese}

Das Prostatakarzinom entsteht aus den Basalzellen des Epithels der Ductuli [18,34]. Mögliche Ursachen sind in der Genetik und in der Immunhistologie $\mathrm{zu}$ suchen $[22,23,32,33,34,51,52]$.

Prostatakarzinome sind lokal aggressiv und haben eine hohe Neigung zur Invasion in die Urethra, die Harnblase oder das umgebende Weichteilgewebe $[18,44]$. Bei bis zu 64-80\% der Tiere liegt zum
Zeitpunkt der Sektion, der häufig gleichzeitig auch der Zeitpunkt der Diagnosestellung ist, eine Metastasierung vor [13, 51]. Prostatakarzinome metastasieren am häufigsten in die Sublumballymphknoten (43\% [51], 64\% [13]), die Lunge (32\% [51], 62\% [13]) und in das Skelettsystem (25,5\% [51], 28\% [13]). Dabei sind Knochenmetastasen vor allem in den Lendenwirbeln und dem Becken zu finden $[13,43,51]$. Die Knochen der Gliedmaßen sind seltener betroffen $[8,13,17,35]$. Außerdem wurden seltener auch Metastasen in der Leber, Niere, Milz, im Colon, in den Nebennieren sowie im Herz und Gehirn nachgewiesen [13,21,44,51].

\section{Klinik}

Zum Zeitpunkt der Diagnosestellung zeigen die Tiere bereits meist eine deutliche Symptomatik [44]. Neben harnwegsspezifischen Symptomen wie Dysurie, Strangurie, Hämaturie oder auch Harnträufeln $[13,18,31,43,50,54]$ zeigen die Tiere unspezifische Symptome wie Gewichtsverlust, Anorexie, Bewegungsunlust und eine allgemeine Schmerzhaftigkeit $[13,18,31,43,44,54]$. In der Literatur wird beschrieben, dass bei betroffenen Tieren aufgrund der vergrößerten Prostata und/oder vergrößerten Sublumballymphknoten Tenesmus beobachtet werden kann $[13,18,50,54]$. Dies können die Autoren nur in Ausnahmefällen bestätigen.

\section{konkret}

Die betroffenen Rüden werden in der Regel aufgrund von Harnabsatzproblemen vorgestellt.

Die Prostata ist trotz der neoplastischen Veränderungen meist nur ggr. vergrö- 
ßert. Das für sonstige Neoplasien typische Größenwachstum tritt beim Prostatakarzinom typischerweise nicht auf, wobei dazu keine konkreten Zahlen vorliegen. Auch die sublumbalen Veränderungen alleine reichen meist nicht aus, damit es zu Kotabsatzproblemen kommt.

\section{Diagnostik}

\section{Rektale Untersuchung}

Neben der allgemeinen Untersuchung wird eine rektale Untersuchung durchgeführt $[11,18,44,50,56]$. Dabei werden Größe, Symmetrie, Oberfläche und Beweglichkeit der Prostata beurteilt $[11,18$, $44,50,56]$. Ein weiteres wichtiges Kriterium, das in der Literatur beschrieben wird, ist die Schmerzhaftigkeit bei Palpation der Prostata $[18,50]$. Dieser Palpationsschmerz bei der rektalen Untersuchung ist aber nicht zwingend hinweisend. Es gibt eine nicht unerhebliche Anzahl an Patienten, die trotz Prostatakarzinom nicht schmerzhaft reagieren. Im Allgemeinen ist das Parenchym bei der rektalen Untersuchung außergewöhnlich derb.

\section{Sonografische Untersuchung}

Die sonografische Untersuchung der Prostata dient als Standardtechnik für die Beurteilung des Organs und liefert viele nützliche Informationen $[19,44,50]$. Jedoch kann mittels Ultraschalls nicht sicher zwischen benignen und malignen Prostataerkrankungen unterschieden werden $[19,41,50]$. Häufig kann jedoch eine Verdachtsdiagnose erhoben werden. Dennoch ist insbesondere die Differenzierung zwischen einem Prostatakarzinom und einer chronischen Prostataentzündung meist nicht möglich.

\section{Physiologische Prostata}

Die Prostata liegt im kaudalen Abdomen, kaudal der Blase bzw. je nach Füllungszustand der Blase im Bereich des kranialen Beckeneingangs [28]. Dorsal der Prostata befindet sich das Colon descendens bzw. das Rektum [28,50]. Die genaue Lokalisation, die Größe und das sonografische Bild der Prostata variieren mit dem Alter des Rüden, eventuellen Vorerkrankungen des Organs und dem Kastrationsstatus $[20,28,29,41]$. Ebenso spielen die Einstellungen des Ultraschallgeräts und die Frequenzen, mit denen das Organ untersucht wird, eine entscheidende Rolle [41].

Die Prostata eines unkastrierten Rüden ist im Längsschnitt rund bis ovoid [28,41] ( Abb.1). Im Querschnitt besteht die Prostata aus 2 großen, symmetrischen Anteilen, die in der Mitte durch eine vertikal verlaufende Naht und der prostatischen Urethra (mit umgebenden M. urethralis) getrennt sind $[28,41]$ ( $\triangleright$ Abb.2). Das Prostataparenchym ist von mittlerer Echogenität, relativ homogen und besitzt eine feine bis mittlere grobkörnige Echotextur [41]. Umgeben wird die Prostata von einer glatten Kapsel [41], die jedoch im Ultraschall nicht immer sichtbar ist. Mit zunehmendem Alter (bereits ab 3 Jahren) des Rüden verändert sich auch das sonografische Bild der Prostata [28]. Es kommt zu einer Größenzunahme des Organs und gesteigerten Echogenität des Prostataparenchyms [28]. In der Literatur wird eine signifikante Korrelation der Prostatagröße mit dem Alter und dem Körpergewicht beschrieben $[5,48]$. Hunde ab 4 Jahren können Veränderungen im Sinne einer benignen Prostatahyperplasie zeigen [41]. Die Prostata ist hierbei meist deutlich vergrößert und besitzt ein inhomogenes Parenchym mit einer variablen Echogenität [41]. Diese Prostataveränderung ist meist ein $\mathrm{Zu}$ fallsbefund, kann jedoch aufgrund der deutlichen Größenzunahme des Organs auch Probleme beim Harn- und Kotabsatz verursachen [41]. Intraparenchymatöse Zysten in variabler Größe und unterschiedlicher Anzahl sind möglich [41], Mineralisationen sind untypisch [41]. Abgesehen von einer benignen Prostatahyperplasie können gerade beim älteren Hund auch einzelne Prostatazysten als Zufallsbefund gefunden werden [48]. Benigne Veränderungen der Prostata wie die benigne Prostatahyperplasie entzündliche Erkrankungen oder Prostatazysten, kommen häufiger als Neoplasien vor $[28,31,49,55]$.

Im Vergleich zum unkastrierten Rüden ist die Prostata eines kastrierten Hundes klein und schlecht von der Umgebung

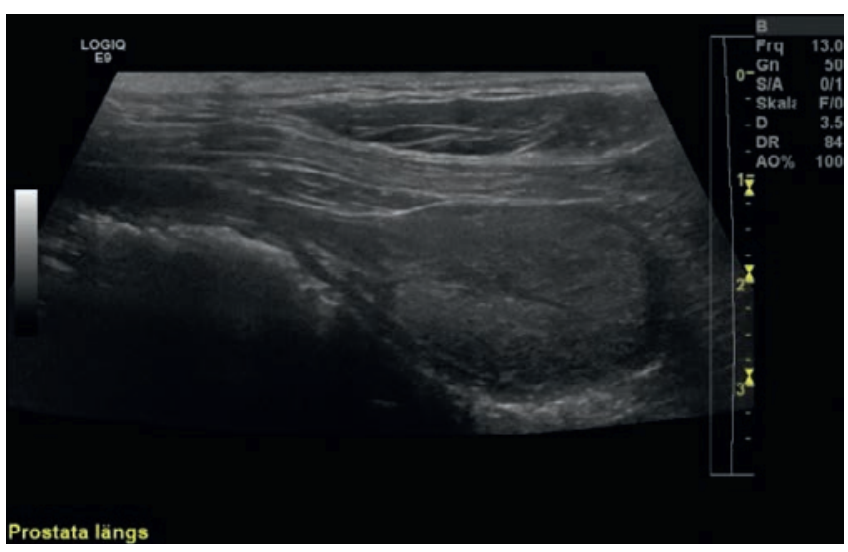

Abb. 1 Sonografisches Bild der physiologischen Prostata eines unkastrierten Rüden im Längsschnitt. Das Organ ist rund bis ovoid und von einer dünnen, hyperechogenen Kapsel umgeben. Das Parenchym ist von mittlerer Echogenität und besitzt eine feine, grobkörnige Echotextur. Dorsal der Blase ist das gefüllte Colon descendens sichtbar. Nach kranial ist die Urethra bis zum Blasenhals verfolgbar. (c) F. Eberhardt



Abb. 2 Sonografischer Querschnitt der Prostata eines unkastrierten Rüden. Das Organ besteht aus 2 großen, symmetrischen Anteilen, die in der Mitte durch eine vertikal verlaufende Naht und die prostatische Urethra (mit umgebenden M. urethralis) getrennt sind. Ventral der Prostata ist das Colon descendens im Querschnitt sichtbar. (c) F. Eberhardt 


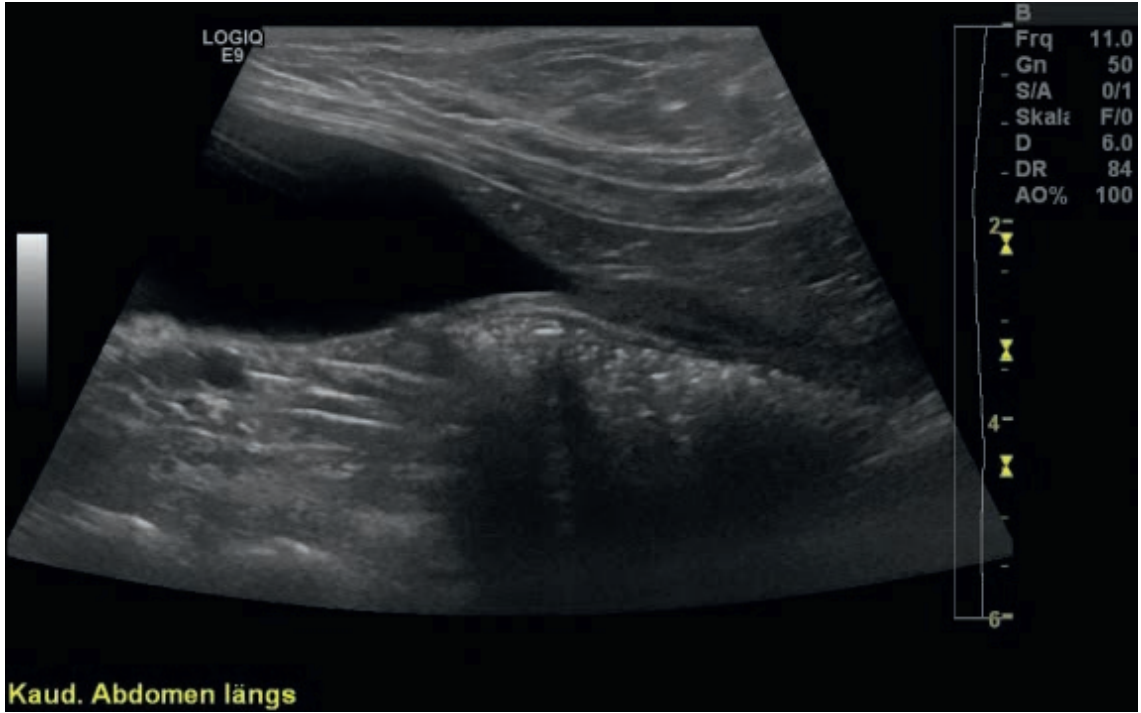

Abb. 3 Sonografisches Bild der Prostata eines kastrierten Rüden. Die Prostata ist im Vergleich zum unkastrierten Rüden ( Abb. 1) klein und spindelförmig. Das Parenchym ist nahezu homogen hypoechogen und die beiden Prostataanteile können nicht mehr voneinander unterschieden werden. (c) F. Eberhardt

abgrenzbar $[28,41]$. Das Parenchym ist homogen hypoechogen und die beiden Prostataanteile können sonografisch nicht mehr voneinander unterschieden werden $[28,41]$, weshalb die Prostata ein eher spindelförmiges Aussehen annimmt ( $\triangleright$ Abb.3). Bei älteren kastrierten Rüden können hin und wieder Inhomogenitäten im Parenchym beobachtet werden [28].

\section{Neoplastische Veränderungen der Prostata}

Das sonografische Bild einer maligne veränderten Prostata ist variabel $[28,41]$. Durch die maligne Infiltration der Prostata kann es zu einer Vergrößerung des Organs kommen $[28,41]$, jedoch ist die Vergrößerung in der Regel nicht so stark ausgeprägt, wie es bei einer benignen Prostatahyperplasie der Fall ist. Aus Sicht der Autoren besteht - wenn überhaupt eine marginale Vergrößerung des Organs. Die Organoberfläche erscheint irregulär und die Echotextur ist heterogen verändert $[8,19,28,41]$. Im Transversalanschnitt können die beiden Prostataanteile unsymmetrisch erscheinen $[8,19,28]$. Oft werden fokale Mineralisationen im Organparenchym beobachtet $[8,9,19,28,41,50,62]$ ( Abb.4). Hierbei ist allerdings $\mathrm{zu}$ beachten, dass dystrophe Verkalkungen auch bei chronischen Prostatitiden vorkommen können $[28,41]$. Bei einer Prostatitis sind hyperechogene
Areale im Gewebe sichtbar [41] ( $\triangleright$ Abb.5). Diese können Ausdruck einer Fibrose, Mineralisationen oder Gasbläschen sein [41].

\section{konkret}

Allein mittels sonografischer Untersuchung ist es schwierig, zwischen einer Neoplasie und einer Prostatitis zu unterscheiden.

Eine Neoplasie und eine Prostatitis können auch parallel auftreten $[41,50]$. Einer Studie zufolge [9] sprechen Mineralisationen im Prostatagewebe bei kastrierten Rüden mit Sicherheit für eine Neoplasie (positiver prädiktiver Wert 100\%). Hinweise auf eine Neoplasie liefern zudem Veränderungen in der Urethra, dem Harnblasenhals und der Blase $[8,19,41]$. Aufgrund von Veränderungen im Bereich des Trigonums der Blase kann es sekundär zu einem Stau des Ureters (Hydroureter) bis hin zu einer Hydronephrose kommen [41]. Weiterhin können die Sublumballymphknoten vergrößert und verändert sein $[18,28,41,44]$. Da diese auch bei einer Prostatitis vergrößert sein können, wird bei entsprechendem Verdacht eine Probenentnahme aus der Prostata und den veränderten Lymphknoten empfohlen, um eine definitive Diagnose stellen zu können [41].

\section{Röntgenologische Untersuchung}

Eine seitliche Übersichtsaufnahme des Abdomens bzw. des Beckens liefert im Vergleich zur Sonografie deutlich weniger Informationen über eine mögliche Erkrankung der Prostata $[44,50]$. Heutzutage haben die Ultraschallgeräte eine so gute Auflösung, dass die Projektionsradiografie nicht mehr zur Primärdiagnostik der Prostata gehört. Vielmehr werden Übersichtsaufnahmen des Abdomens bzw. des Thorax angefertigt, um eine mögliche Metastasierung zu detektieren.

Fokale Verkalkungen im Prostataparenchym können möglicherweise im Röntgenbild sichtbar sein $[9,18,44]$. Diese können beim unkastrierten Rüden als Folge einer schweren Prostatitis oder Prostatazysten auftreten [9]. Allein dieser Befund lässt beim intakten Rüden keinen Rückschluss auf die Dignität zu (Sensitivität: 67\%, Spezifität: 77\%, positiver prädiktiver Wert: 22\% [9]). Beim kastrierten Rüden hingegen sind fokale Verkalkungen im Prostataparenchym sehr hinweisend auf eine Neoplasie des Organs (Sensitivität: $84 \%$, Spezifität: $100 \%$, positiver prädiktiver Wert: 100\% [9]) ( A Abb.6). Wesentlich hilfreicher zur Prostatabeurteilung sind Röntgenaufnahmen zur Detektion von möglichen tumorspezifischen Knochenveränderungen und Metastasen in anderen Organen (z.B. der Lunge) [44]. Bis zu 45\% der betroffenen Rüden können im Röntgen Hinweise auf Knochenmetastasen haben $[13,43]$. Diese sind im Zusammenhang mit dem Vorbericht und der klinischen Untersuchung annähernd pathognomonisch $[43,44]$. Im Bereich der Lendenwirbelsäule und des Beckens sind hierbei osteolytische oder osteoblastische Veränderungen zu finden $[12,17,42]$. Außerdem können periostale Zubildungen ventral an den kaudalen Lendenwirbeln zu sehen sein $[9,12$, 17,42] ( Abb.7). Eine auf dem Röntgenbild sichtbare Vergrößerung der sublumbalen Lymphknoten ist möglich $[9,18$, 56]. Außerdem sollten Röntgenaufnahmen des Thorax angefertigt werden, um Lungenmetastasen detektieren zu können [18]. In der Studie von Sorenmo et al. [51] zeigten $32 \%$ der Tiere $(n=70)$ mit einem Prostatakarzinom eine Metastasierung in die Lunge, bei Cornell et al. [13] waren es sogar $62 \%$ der Rüden $(n=76)$. 


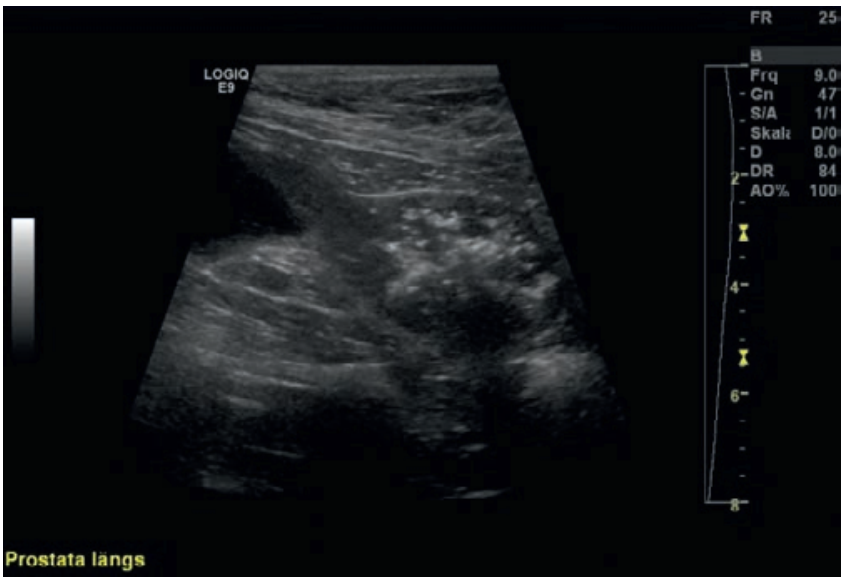

Abb. 4 Sonografisches Bild einer maligne veränderten Prostata bei einem kastrierten Rüden. Das Organ ist größer als eine physiologische Prostata bei einem kastrierten Rüden. Das Gewebe ist hypoechogen und diffus mit Mineralisationen durchsetzt, die einen distalen Schallschatten zeigen. Nach kranial ist der Beginn der Blase sichtbar. (c) F. Eberhardt



Abb. 5 Sonografisches Bild einer chronischen Prostatitis. Das Prostataparenchym ist inhomogen und diffus von kleinen punkt- bis strichförmigen, hyperechogenen Arealen durchsetzt. Aufgrund der Größe dieser hyperechogenen Areale ist nicht mit einem distalen Schallschatten zu rechnen, sollte es sich hierbei um Mineralisationen handeln. Daher könnten diese Veränderungen bindegewebige Einlagerungen oder Verkalkungen im Parenchym sein. Weiterhin ist eine unregelmäßige, längsovale, anechogene Struktur im Prostataparenchym sichtbar. Es handelt sich hierbei um eine intraprostatische Zyste. (c) F. Eberhardt

\section{Weiterführende diagnostische Verfahren}

Schnittbildverfahren wie die Computertomografie (CT) oder die Magnetresonanztomografie (MRT) sind in der Regel nicht nötig, um die Diagnose einer Prostataneoplasie stellen zu können. In der CT können die spezifischen Knochenproliferationen an der Lendenwirbelsäule und dem Becken dargestellt und in ihrem Ausmaß beurteilt werden ( Abb.8). Zudem können metastatische Verkalkungen im umgebenden Weichteilgewebe und den Sublumballymphknoten sehr gut zu sehen sein ( $\triangleright$ Abb.9). In der MRT kann vor allem die Homogenität und Intensität des Prostataparenchyms detektiert werden. Auch hier ist eine sehr gute Beurteilung des umliegenden Weichteilgewebes und der Lymphknoten möglich, jedoch können die spezifischen Knochenveränderungen eher unterschätzt werden ( $\bullet$ Abb. 10).

In der Literatur wird die Möglichkeit beschrieben, mithilfe einer retrograden Röntgenkontrastuntersuchung der Urethra zwischen einem Prostatakarzinom und einem Übergangsepithelkarzinom zu unterscheiden [43,44,57]. Jedoch konnten andere Autoren zeigen, dass der Kontrastmittelreflux in das Prostataparenchym keinen Rückschluss auf die Dig- nität der Prostataveränderung zulässt [1] Da diese Methode wenig spezifisch ist, wird sie in der Praxis nicht angewendet.

\section{Zytologische Untersuchung}

Die letztendliche Diagnosesicherung erfolgt über den Nachweis von Tumorzellen $[18,50,54]$. Zellen für die zytologische Untersuchung können mittels

- Feinnadelaspiration (ultraschallgestützt)

- Bürstentechnik

- Kathetersaugbiopsie

gewonnen werden $[30,43,47,54,61]$.

Die ultraschallgestützte Feinnadelaspiration ist einfach durchzuführen und birgt nur ein sehr geringes Risiko für den Patienten [44,54]. Einer Studie zufolge [47] zeigt die Feinnadelaspiration eine 75\%ige Übereinstimmung mit der Histopathologie. In der Literatur wird lediglich von wenigen Fällen berichtet, bei denen es bei einer Feinnadelaspiration zu einer Tumorzelltransplantation in den Stichkanal der Nadel gekommen ist [45]. Allerdings waren die Primärtumoren Übergangsepithelkarzinome [45]. Die Autoren können in 15 Jahren Klinikerfahrung von keinem Fall berichten, bei dem nach einer Punktion oder Biopsie eine Tumorzelltransplantation nachgewiesen wurde. Auch konnte keine bakterielle Streuung nach einer Punktion der Pros- tata (bakterielle Prostatitis, Prostatazysten, Prostataabszess) nachgewiesen werden. Daher wird das Risiko für eine Tumorzelltransplantation oder bakterielle Streuung in den Stichkanal als äußerst gering eingeschätzt.

Die Kathetersaugbiopsie ist kostengünstig, technisch einfach und ohne Ultraschallkontrolle durchzuführen [44,54], jedoch kann sie gelegentlich zu wenig Zellmaterial enthalten [44,54]. Zusammen mit der Feinnadelaspiration haben die Proben der Kathetersaugbiopsie in der zytologischen Untersuchung von 27 Hunden mit einem Prostatakarzinom eine Sensitivität von $73 \%$ und eine Spezifität von $98 \%$ (positiver prädiktiver Wert: 95\%, negativer prädiktiver Wert: $87 \%$ [54]). Aus Sicht der Autoren wird dieses Verfahren praktisch nicht mehr angewendet. Aufgrund der sonografisch gestützten, gezielten Probenentnahme aus dem Prostataparenchym mittels Feinnadelaspiration und/oder Biopsie kann sicher und ausreichend viel Material für die Beurteilung gewonnen werden.

\section{Histologische Untersuchung}

Die Prostatabiopsie ist aus Sicht der Autoren das sicherste Nachweisverfahren. Mit dieser kann genügend Material für eine Histologie gewonnen werden 


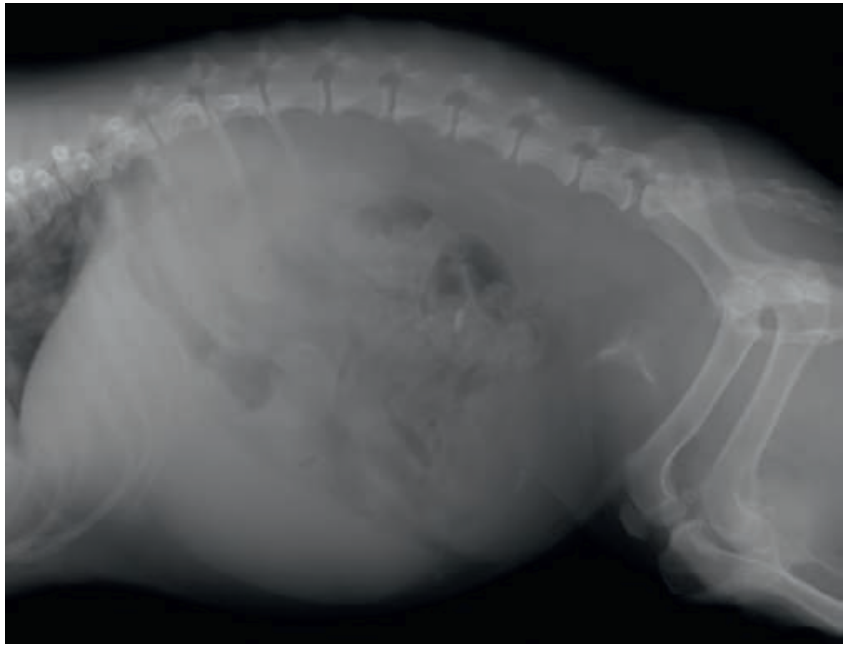

Abb. 6 Röntgenbild des Abdomens eines Rüden im laterolateralen Strahlengang. Der Harnblasenschatten ist weit intraabdominal gelegen. Zwischen der Blase und dem Becken ist ein kleinerer, längsovaler Weichteilschatten erkennbar, der strichförmige Mineralisationen zeigt. Es handelt sich hierbei um die deutlich vergrößerte Prostata, die multifokale Mineralisationen im Parenchym aufweist. Diese Veränderungen im Röntgenbild sind mit der entsprechenden Klinik bei einem kastrierten Rüden sehr hinweisend auf eine Prostataneoplasie. @ F F. Eberhardt

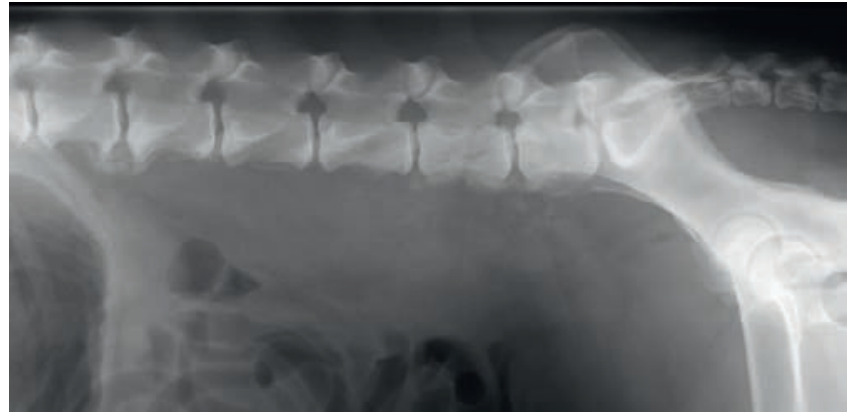

Abb. 7 Röntgenbild der Lendenwirbelsäule eines kastrierten Rüden im laterolateralen Strahlengang. Im Bereich von L5-L7 sind deutliche periostale Zubildungen ventral am Wirbelkörper zu sehen. Weiterhin ist ventral der Lendenwirbelsäule ein vergrößerter Weichteilschatten sichtbar, der dezent angedeutete Verkalkungen zeigt. Dies ist hinweisend auf vergrößerte Sublumballymphknoten und/oder eine Infiltration in das umliegende Weichteilgewebe. ( ) F. Eberhardt
( Abb.11, $\triangleright$ Abb. 12). Hierbei ist zu beachten, dass sich die Biopsie aufgrund der meist multifokalen Verkalkungen im Prostatagewebe als relativ schwierig und

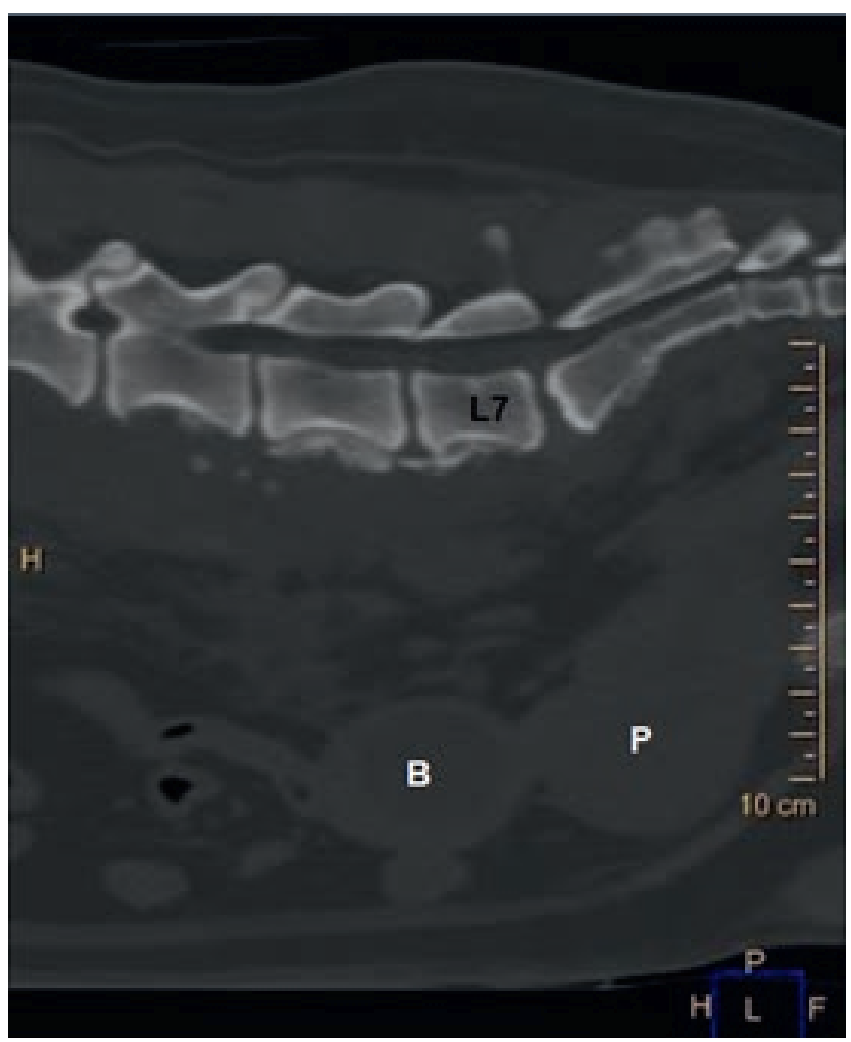

Abb. 8 Computertomografischer Sagittalschnitt durch das kaudale Abdomen eines kastrierten Rüden. Zu sehen sind deutliche periostale Zubildungen ventral an den Wirbelkörpern der kaudalen Lendenwirbelsäule sowie ventral am Sakrum. Ventral der Lendenwirbelsäule ist ein deutlich vergrößerter Weichteilschatten sichtbar, der kleine, rundliche, mineraldense Areale zeigt. Es handelt sich hierbei um vergrößerte Sublumballymphknoten bzw. ist eine Infiltration in das umliegende Weichteilgewebe möglich. Weiter ventral befindet sich die Harnblase (B) und die deutlich vergrößerte Prostata $(P)$. (C) F. Eberhardt tens 4 verschiedenen Stellen Proben genommen werden, da ansonsten die Gefahr besteht, die veränderten Areale der Prostata zufällig nicht zu treffen. Damit ist die Probenanzahl deutlich geringer als in der Humanmedizin: Hier werden 10-12 Biopsieproben entnommen [15]. Um die Urethra während der Biopsie zu schützen, kann zuvor ein Harnkatheter gelegt werden $(\triangleright$ Abb. 13). Dadurch ist der genaue Verlauf der Urethra sichtbar und Verletzungen können vermieden werden.

\section{.konkret \\ In der Urinuntersuchung sind nur selten Tumorzellen zu finden [7], daher ist diese zum Nachweis eines Prostata- karzinoms ungeeignet.}

\section{Therapie und Prognose}

Häufig sind bereits zum Zeitpunkt der Diagnosestellung Metastasen vorhanden $[13,44,51]$. Aufgrund der meist hochgradigen Schmerzen der Tiere, die vor allem durch die Knochenmetastasen verursacht werden, entscheiden sich die Besitzer an diesem Punkt zur Euthanasie $[13,44]$. Eine mögliche palliative Therapie ist je nach Klinik und Metastasierung 


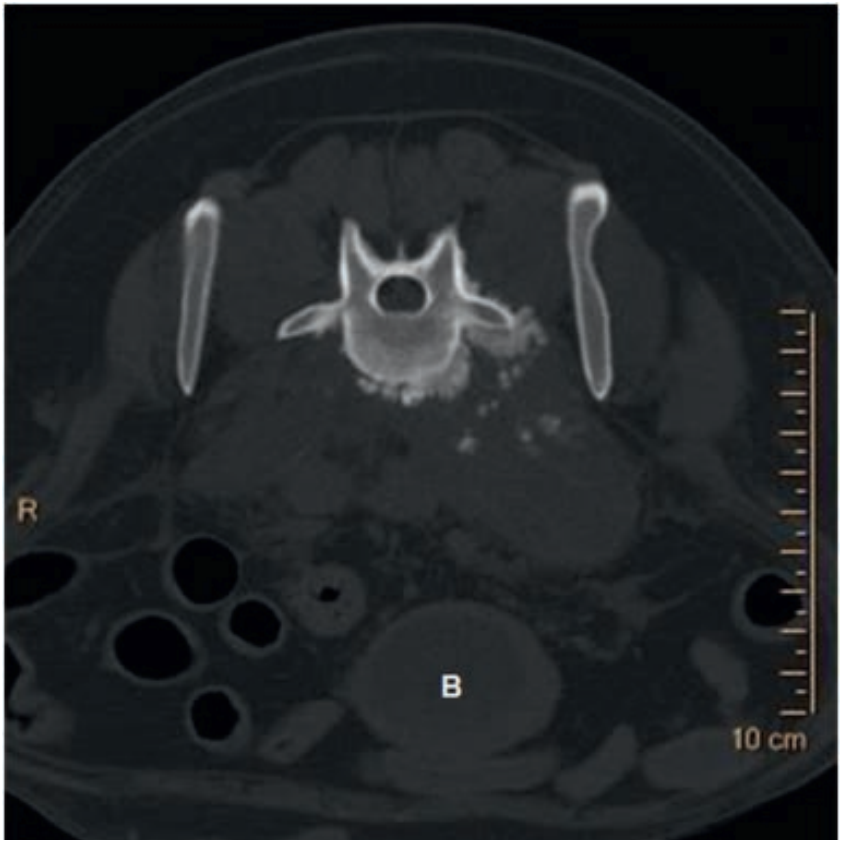

Abb. 9 Computertomografischer Transversalschnitt durch das kaudale Abdomen eines kastrierten Rüden. Zu sehen sind deutliche periostale Zubildungen an den Wirbelkörpern und den Transversalfortsätzen. Ventrolateral des Wirbels ist ein deutlich vergrößerter Weichteilschatten sichtbar, der kleine, rundliche, mineraldense Areale zeigt. Es handelt sich hierbei um vergrößerte Sublumballymphknoten bzw. ist eine Infiltration in das umliegende Weichteilgewebe möglich. Weiter ventral befindet sich die Harnblase (B). (C) F. Eberhardt

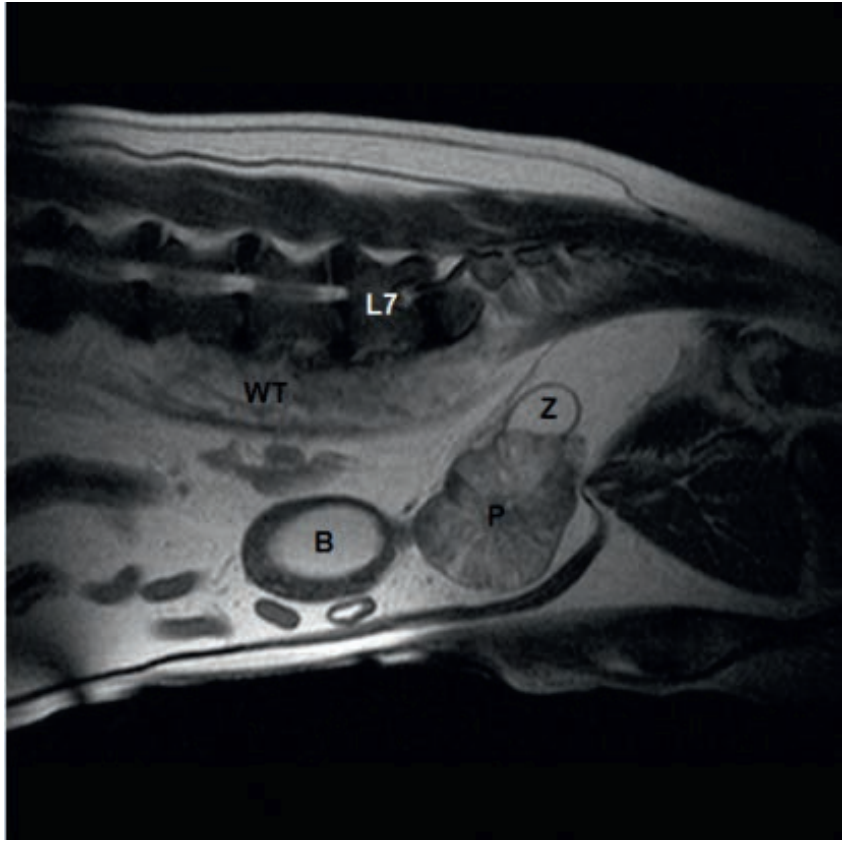

Abb. 10 T2-gewichteter Sagittalschnitt des kaudalen Abdomens eines kastrierten Rüden in der MRT. Die Prostata (P) liegt kaudal der Harnblase (B) und ist für einen kastrierten Rüden deutlich vergrößert. Das Organ zeigt eine irreguläre Oberfläche und das Prostataparenchym ist inhomogen. Kaudodorsal geht eine zystische Struktur (Z) aus der Prostata hervor. Ventral der kaudalen Lendenwirbelsäule (L7) ist ein vergrößerter Weichteilschatten erkennbar (WT). (c) F. Eberhardt in der Literatur beschrieben $[8,16,24,37$, $38,39,40,50,52,58,59]$. Jedoch beträgt die Überlebenszeit lediglich einige Tage bis mehrere Monate $[8,13,24,37,38,39$, $40,52,58,59,60]$. Dadurch, dass die meisten betroffenen Rüden kastriert sind, spielt die Kastration keine Rolle mehr.
Bei unkastrierten Rüden hat die

Kastration keine Auswirkungen auf den Erkrankungsverlauf [8], ebenso so wenig wie die Gabe von Antiandrogenen $[8,50]$.

Eine Chemotherapie ist in Einzelfällen beschrieben [16,27], führte jedoch nicht zur Heilung der Patienten. Einer Studie

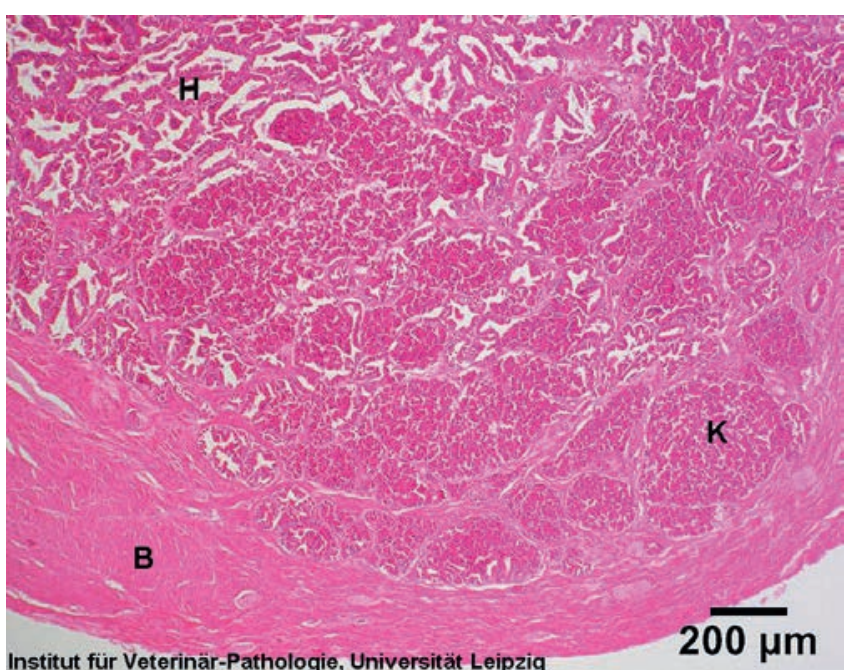

Abb. 11 Prostatahyperplasie, Übergang zum Prostatakarzinom. In Bereichen mit einer Prostatahyperplasie $(\mathrm{H})$ zeigt sich eine papilliforme Proliferation eines regulär differenzierten und strukturierten Drüsenepithels, wobei alle papilliformen Projektionen einen stromalen Grundstock besitzen. Dagegen füllen in Bereichen mit einem Prostatakarzinom (K) die neoplastischen Epithelzellen die glandulären Strukturen nahezu vollständig aus, stromale Anteile sind in den Projektionen nicht erkennbar. Fibromuskuläres Stroma (B), H.-E.-Färbung. @ Frau Dr. Müller, Institut für Veterinär-Pathologie, Universität Leipzig zufolge [52] überlebten Tiere mit Piroxicam oder Carprofen signifikant länger als unbehandelte Rüden (6,9 Monate bzw. 0,7 Monate). Andere Autoren konnten eine deutliche klinische Verbesserung der Tiere unter Meloxicam beobachten [44].

Die Bestrahlung wurde vereinzelt eingesetzt $[3,8,58]$, jedoch zeigten die Tiere deutliche Nebenwirkungen [3] und je nach Studie und möglicher Metastasierung Überlebenszeiten von 80-180 Tagen [8,58].

In einer Studie mit 10 Rüden mit einem Prostatakarzinom führte die komplette Prostatektomie bei allen Patienten zur Inkontinenz und einer Überlebenszeit von durchschnittlich unter 20 Tagen [59]. Hingegen hatten 10 Rüden mit einer subtotalen Prostatektomie (Erhalt der dorsalen Prostatakapsel und der Urethra) eine durchschnittliche Überlebenszeit von 112 Tagen [59]. Wenn es aufgrund der Infiltration der Urethra oder des Trigonums der Blase zu einer Obstruktion gekommen ist, können palliative Maßnahmen wie das Legen eines Harnkatheters oder 

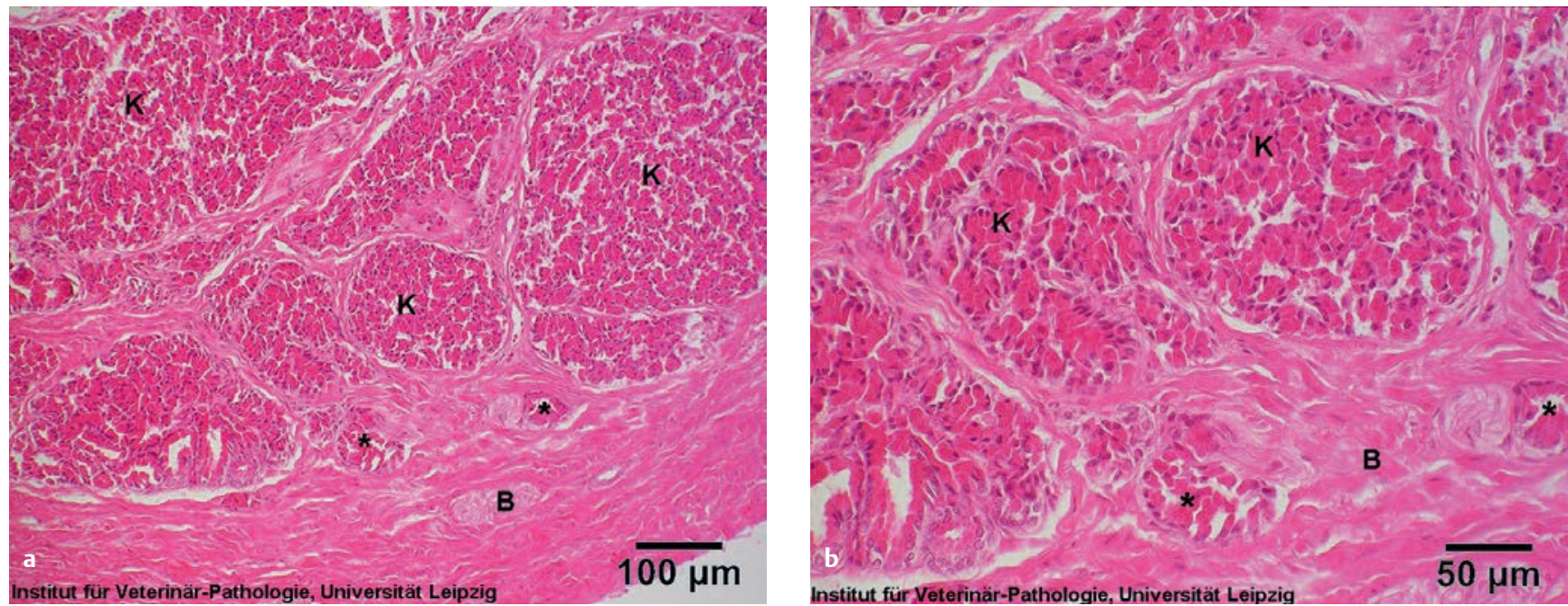

Abb. 12 Prostatakarzinom. Innerhalb des Prostatakarzinoms (K) füllen die neoplastischen, geringgradig ungleichmäßig differenzierten Epithelzellen die glandulären Strukturen nahezu vollständig aus, wobei stromale Anteile in den Projektionen nicht erkennbar sind. Multifokal ist ein infiltratives Wachstum ${ }^{*}$ ) in das fibromuskuläre Stroma (B) erkennbar, H.-E.-Färbung. @ @ Frau Dr. Müller, Institut für Veterinär-Pathologie, Universität Leipzig

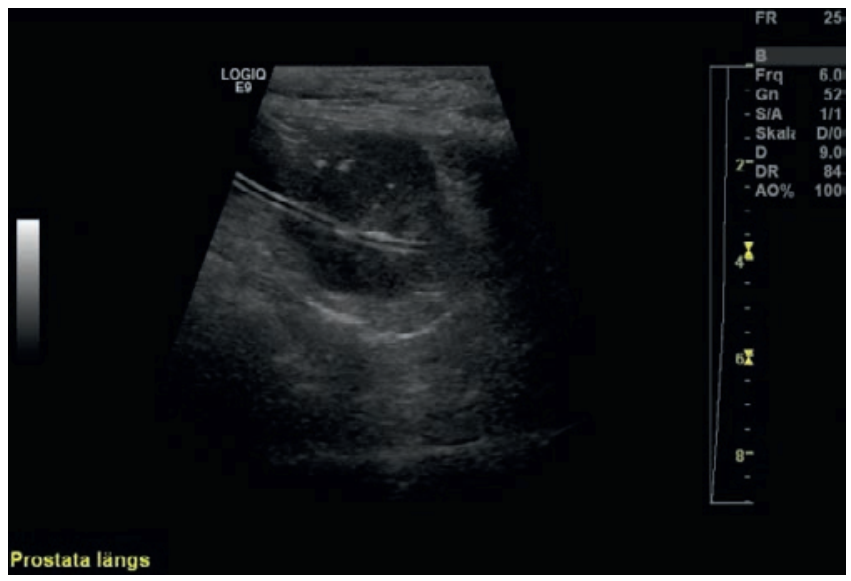

Prostata gewonnen werden. @ F. Eberhardt

eines Urethrastents zumindest vorübergehend den Harnabsatz gewährleisten.

Insgesamt ist festzustellen, dass es keine kurative Therapie für das kanine Prostatakarzinom gibt. Je nach Schweregrad der Erkrankung, der klinischen Symptome und einer möglichen Metastasierung ergibt sich eine infauste Prognose für Rüden mit einem Prostatakarzinom.

\section{Fazit für die Praxis}

Auch wenn das Prostatakarzinom des Hundes selten ist, stellt es eine ernst $\mathrm{zu}$ nehmende und relativ schnell progressiv verlaufende Erkrankung dar. Vor allem kastrierte Rüden fortgeschrittenen Alters sind prädisponiert. Wenn Verände-
Abb. 13 Sonografisches Bild einer vergrößerten Prostata bei einem kastrierten Rüden. Das Parenchym ist hypoechogen inhomogen und es sind rundliche, reflexreiche Areale (fokale Mineralisationen) erkennbar. Der Harnkatheter ist als reflexreiche Doppellammelle in der Urethra erkennbar. Mithilfe des Harnkatheters können unter Schutz der Urethra Proben aus der rungen der Prostata oder der Urethra nicht als Zufallsbefund während einer sonografischen Untersuchung des Abdomens gefunden werden, sind die betroffenen Tiere meist in einem fortgeschrittenen Erkrankungsstadium und die Wahrscheinlichkeit der Metastasierung ist hoch. Die Probenentnahme mittels sonografisch gestützter Biopsie aus mindestens 4 Stellen ist das Mittel der Wahl für die Diagnosestellung. Wenn überhaupt gewünscht oder vertretbar, ist nur noch eine palliative Therapie möglich, um den Tieren zu helfen. Die Prognose ist infaust.

\section{Online zu finden unter}

http://dx.doi.org/10.1055/s-0035-1550134

\section{Literatur}

1 Ackerman N. Prostatic reflux during positive contrast retrograde urethrography in the dog. Vet Rad 1983; 24: 251-259

2 Alizadeh M, Alizadeh S. Survey of clinical and pathological characteristics and outcomes of patients with prostate cancer. Glob J Health Sci 2014; 6: 49-57

3 Anderson CR, McNiel EA, Gillette EL et al. Late complications of pelvic irradiation in 16 dogs. Vet Radiol Ultrasound 2002; 43: 187-192

4 Assin R, Balsi A, Citro G, Spugnini EP. Prostate as sole unusual recurrence site of lymphoma in a dog. In Vivo 2008; 22: 755-757

5 Atalan G, Holt PE, Barr FJ. Ultrasonographic estimation of prostate size in normal dogs and relationship to bodyweight and age. J Small Anim Pract 1999; 40: 119-122

6 Bacci B, Vignoli M, Rossi F et al. Primary prostatic leiomyosarcoma with pulmonary metastases in a dog. J Am Anim Hosp Assoc 2010; 46: 103-106

7 Barsanti JA, Finco DR. Evaluation of techniques for diagnosis of canine prostatic diseases. J Am Vet Med Assoc 1984; 185: 198-200

8 Bell FW, Klausner JS, Hayden DW et al. Clinical and pathologic features of prostatic adenocarcinoma in sexually intact and castrated dogs: 31 cases (1970-1987). J Am Vet Med Assoc 1991; 199: 1623-1630

9 Bradbury CA, Westropp JL, Pollard RE. Relationship between prostatomegaly, prostatic mineralization, and cytologic diagnosis. Vet Radiol Ultrasound 2009; 50: 167-171 
10 Bryan JN, Keeler MR, Henry CJ et al. A population study of neutering status as a risk factor for canine prostate cancer. Prostate 2007; 67: 1174-1181

11 Chun R, Garrett LD. Urogenital and mammary gland tumors. In: Ettinger $\mathrm{S}$, Feldman EC, Hrsg. Textbook of Veterinary Internal Medicine. St. Louis, Missouri: Saunders WB; 2010: 2208-2212

12 Cooley DM, Waters DJ. Skeletal metastasis as the initial clinical manifestation of metastatic carcinoma in 19 dogs. J Vet Intern Med 1998; 12: $288-293$

13 Cornell KK, Bostwick DG, Cooley DM et al. Clinical and pathologic aspects of spontaneous canine prostate carcinoma: a retrospective analysis of 76 cases. Prostate 2000; 45 : 173-183

14 Della Santa D, Dandrieux J, Psalla D et al. Primary prostatic haemangiosarcoma causing severe haematuria in a dog. J Small Anim Pract 2008; 49: 249-251

15 Deutsche Krebsgesellschaft. Yiallouros M, Christmann D. Prostatakrebs, Krebs der Vorsteherdrüse (10.09.2014). Im Internet: http://www.krebsgesellschaft.de/onko-internetportal/basis-informationen-krebs/ krebsarten/prostatakrebs/definition-und-haeufigkeit.html; Stand: 28.02.2015

16 Dominguez PA, Dervisis NG, Cadile CD et al. Combined gemcitabine and carboplatin therapy for carcinomas in dogs. J Vet Intern Med 2009; 23: 130-137

17 Durham SK, Dietze AE. Prostatic adenocarcinoma with and without metastasis to the bone in dogs. J Am Vet Med Assoc 1986; 188: 1432-1436

18 Fan TM, de Lorimier LP. Tumors of the male reproductive system. In: Withrow SJ, Vail DM,
Hrsg. Withrow \& MacEwen's Small Animal Clinical Oncology. St. Louis, lowa: Saunders (Elsevier); 2007: 637-648

19 Feeney DA, Johnston GR, Klausner JS et al. Canine prostatic disease-comparison of ultrasonographic appearance with morphologic and microbiologic findings: 30 cases (1981-1985). J Am Vet Med Assoc 1987; 190: 1027-1034

20 Feeney DA, Johnston GR, Klausner JS, Bell FJ. Canine prostatic ultrasonography: 1989. Semin Vet Med Surg (Small Anim) 1989; 4: 44-57

21 Francey T. Prostatic Diseases. In: Ettinger SJ, Feldman EC, Hrsg. Textbook of Veterinary Internal Medicine. St. Louis, Missouri: Saunders WB; 2010: 2047-2058

22 Gallardo F, Mogas T, Baró T et al. Expression of androgen, oestrogen alpha and beta, and progesterone receptors in the canine prostate: differences between normal, inflamed, hyperplastic and neoplastic glands. J Comp Pathol 2007; 136: 1-8

23 Grieco V, Riccardi E, Rondena M et al. The distribution of oestrogen receptors in normal, hyperplastic and neoplastic canine prostate, as demonstrated immunohistochemically. J Comp Pathol 2006; 135: 11-16

24 Hardie EM, Barsanti JA, Rawlings CA. Complications of prostatic surgery. J Am Hosp Assoc 1984; 20: 50-56

25 Hayden DW, Bartges JW, Bell FW, Klausner JS. Prostatic hemangiosarcoma in a dog: Clinical and pathological findings. J Vet Diagn Invest 1992; 4: 209-211

26 Hayden DW, Klausner JS, Waters DJ. Prostatic leiomyosarcoma in a dog. J Vet Diagn Invest 1999; 11: 283-286
27 Hazzah TN, Brodsky EM, Post GS. Evaluation of mitoxantrone and a COX inhibitor as a first line therapy for canine prostatic carcinoma. Proc $28^{\text {th }}$ Ann Conf Vet Cancer Soc, Seattle 2008; 19

28 Hecht S. Male reproductive tract. In: Penninck D, d’Anjou MA, Hrsg. Atlas of Small Animal Ultrasonography. Awes, lowa: Blackwell; 2008: 417-443

29 Johnston GR, Feeney DA, Rivers B, Walter PA. Diagnostic imaging of the male canine reproductive organs: Methods and limitations. Vet Clin North Am Small Anim Pract 1991; 21: 553-589

30 Kay ND, Ling GV, Nyland TG et al. Cytological diagnosis of canine prostatic disease using a urethral brush technique. J Am Anim Hosp Assoc 1989; 25: 517-526

31 Krawiec DR, Heflin D. Study of prostatic disease in dogs: 177 cases (1981-1986). J Am Vet Med Assoc 1992; 200: 1119-1122

32 Lai CL, L'Eplattenier H, van den Ham R et al. Androgen receptor CAG repeat polymorphisms in canine prostate cancer. J Vet Intern Med 2008; 22: 1380-1384

33 Lai CL, van den Ham R, van Leenders G et al. Histopathological and immunohistochemical characterization of canine prostate cancer. Prostate 2008; 68: 477-488

34 Leav I, Schelling KH, Adams JY et al. Role of canine basal cells in postnatal prostatic development, induction of hyperplasia, and sex hormone-stimulated growth, and the ductal origin of carcinoma. Prostate 2001; 48: 210-224

35 Lee-Parritz DE, Lamb CR. Prostatic adenocarcinoma with osseous metastases in a dog. J Am Vet Med Assoc 1988; 195 : 1569-1572 
36 Leitlinienprogramm Onkologie (Deutsche Krebsgesellschaft, Deutsche Krebshilfe, AWMF): Interdisziplinäre Leitlinie der Qualität S3 zur Früherkennung, Diagnose und Therapie der verschiedenen Stadien des Prostatakarzinoms, Langversion 3.1 (Oktober 2014). Im Internet: http://leitlinienprogramm-onkologie.de/Leitlinien.7.0.html; Stand: 28.02.2015

37 Liptak JM, Brutscher SP, Monnet E et al. Transurethral resection in the management of urethral and prostatic neoplasia in 6 dogs. Vet Surg 2004; 35: 505-516

38 Lucroy MD, Bowles MH, Higbee RG et al. Photodynamic therapy for prostatic carcinoma in a dog. J Vet Intern Med 2003; 17: 235-237

39 L'Eplattenier HF, van Nimwegen SA, van Sluijs FJ, Kirpensteijn J. Partial prostatectomy using $\mathrm{Nd}$ : YAG laser for management of canine prostate carcinoma. Vet Surg 2006; 35 : 406-411

40 L'Eplattenier HF, Klem B, Teske E et al. Preliminary results of intraoperative photodynamic therapy with 5-aminolevulenic acid in dogs with prostate carcinoma. Vet J 2008; 178: 202-207

41 Mattoon JS, Nyland TG. Prostate and testes. In: Nyland TG, Mattoon JS, Hrsg. Small Animal Diagnostic Ultrasound. Philadelphia: WB Saunders; 2015: 608-633

42 Morgan JP, Ackerman N, Bailey CS, Pool RP. Vertebral tumors in the dog: A clinical, radiologic, and pathologic study of 61 primary and secondary lesions. Vet Rad 1980; 21: 197-212

43 Nickel RF, Teske E. Diagnosis of canine prostatic carcinoma. Tijdschr Diergeneeskd 1992; 117: 325

44 Nickel R. Tumoren der Prostata. In: Kessler M, Hrsg. Kleintieronkologie -Diagnose und Therapie von Tumorerkrankungen bei Hund und Katze. Stuttgart: Enke; 2013: 383-387

45 Nyland TG, Wallack ST, Wisner ER. Needletract implantation following US-guided fine-needle aspiration biopsy of transitional cell carcinoma of the bladder, urethra, and prostate. Vet Radiol Ultrasound 2002; 43: 50-53

46 Pinto da Cunha N, Ghisleni G, Romussi S, Caniatti M. Prostatic sarcomatoid carcinoma in a dog: cytologic and immunohistochemical findings. Vet Clin Pathol 2007; 36: 368-372

47 Powe JR, Canfield PJ, Martin PA. Evaluation of cytologic diagnosis of canine prostatic disorders. Vet Clin Pathol 2004; 33: 150-154

48 Ruel Y, Barthez PY, Mailles A, Begon D. Ultrasonographic evaluation of the prostate in healthy intact dogs. Vet Radiol Ultrasound 1998; 39: 212-216

49 Shafiee R. Shariat A, Khalili S et al. Diagnostic investigations of canine prostatitis incidence together with benign prostate hyperplasia, prostate malignancies, and biochemical recurrence in high-risk prostate cancer as a model for human study. Tumour Biol 2014; DOI 10.1007/s13277-014-2854-4

50 Smith J. Canine prostatic disease: a review of anatomy, pathology, diagnosis, and treatment. Theriogenology 2008; 70 : 375-383

51 Sorenmo KU, Goldschmidt M, Schofer F et al. Immunohistochemical characterization of canine prostatic carcinoma and correlation with castration status and castration time. Vet Comp Oncol 2003; 1: 48-56

52 Sorenmo KU, Goldschmidt MH, Shofer FS et al. Evaluation of cyclooxygenase- 1 and cyclooxygenase-2 expression and the effect of cyclooxygenase inhibitors in canine prostatic carcinoma. Vet Comp Onc 2004; 2: $13-23$

53 Stone EA, Thrall DE, Barber DL. Radiographic interpretation of prostatic disease in the dog. J Am Anim Hosp Assoc 1978; 14: 115-118

54 Teske E, Nickel RF. Zur Aussagekraft der Zytologie bei der Diagnostik des Prostatakarzinoms beim Hund. Kleintierprax 1996; 41: 239-247
55 Teske E, Naan BC, van Dijk EM et al. Canine prostate carcinoma: epidemiological evidence of an increased risk in castrated dogs. Mol Cell Endocrinol 2002; 197: 251-255

56 Thornton GW. Radiographs in the diagnosis of diseases condition of the urinary system. Presented at the American Veterinary Radiology Society Meeting; Indianapolis, Indiana, 10. Januar 1961

57 Ticer JW, Spencer CP, Ackerman N. Positive contrast retrograde urethrography: A useful procedure for evaluating urethral disorders in the dog. Vet Radiol 1980; 21: 2-5

58 Turrel JM. Intraoperative radiotherapy of carcinoma of the prostate in ten dogs. J Am Vet Med Assoc 1987; 190: 48-52

59 Vlasin M, Rauser, Fichtel T, Necas A. Subtotal intracapsular prostatectomy as a useful treatment for advanced-stage prostatic malignancies. J Small Anim Pract 2006; 47: 512-516

60 Weaver AD. Fifteen cases of prostatic carcinoma in the dog. Vet Rec 1981; 109: 71-75

61 Winter MD, Locke JE, Penninck DG. Imaging diagnosis: Urinary obstruction secondary to prostatic lymphoma in a young dog. Vet Radiol Ultrasound 2006; 47: 597-601

62 Zohil AM, Castellano MC. Prepubic and transrectal ultrasonography of the canine prostate: A comparative study. Vet Radiol Ultrasound 1995; 36: 393-396

\section{Franziska Eberhardt \\ Dr. Ingmar Kiefer}

Universität Leipzig

Veterinärmedizinische Fakultät

Klinik für Kleintiere

An der Tierkliniken 23

04103 Leipzig 


\section{Fragebogen}

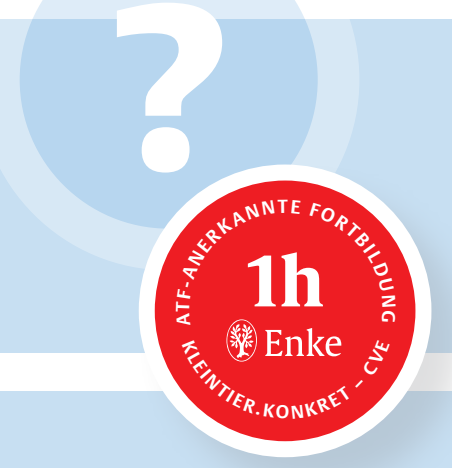

\section{Frage 1}

Das Prostatakarzinom beim Hund ist eine seltene Erkrankung. Wie wird die Prävalenz angegeben?
a. unter $0,1 \%$
b. zwischen $0,2-0,6 \%$
c. zwischen $1-2 \%$
d. zwischen $2-10 \%$
e. zwischen $15-18 \%$

\section{Frage 2}

Kastrierte Rüden erkranken signifikant häufiger am Prostatakarzinom. Um wie viel höher liegt die Wahrscheinlichkeit eines kastrierten Rüden, an einem Prostatakarzinom zu erkranken?
a. 2,1-fach
b. 3,8-fach
c. 5,1-fach
d. $10-$ fach
e. 12 -fach

\section{Frage 3}

Laut einer Untersuchung hat ein Großteil der an einem Prostatakarzinom erkrankten Tiere bereits bei Diagnosestellung nachweisbare Metastasen. Wie hoch liegt der Anteil?
a. $32-40 \%$
b. $44-55 \%$
c. $64-80 \%$
d. $80-85 \%$
e. über $90 \%$

\section{Frage 4}

Ab welchem Alter geht man in der Regel davon aus, dass ein Rüde eine benigne Prostatahyperplasie entwickelt?
a. ab 2 Jahren
b. ab 4 Jahren
c. ab 5 Jahren
d. ab 6 Jahren
e. ab 7 Jahren

\section{Frage 5}

Wie hoch ist der positiv prädiktive Wert in Bezug auf das Vorliegen eines Prostatakarzinoms für Mineralisationen beim kastrierten Rüden?
a. $20 \%$
b. $30 \%$
c. $50 \%$
d. $70 \%$
e. $100 \%$

\section{Frage 6}

Obwohl das Prostatakarzinom nicht primär in die Lunge metastasiert, kommen laut unterschiedlichen Studien auch Metastasen in der Lunge vor. Welche Zahlen werden hierzu angegeben?
a. unter $5 \%$
b. zwischen $6-10 \%$
c. zwischen $11-15 \%$
d. zwischen $18-24 \%$
e. über $30 \%$

\section{Frage 7}

Zur Diagnosesicherung wird neben der Prostatabiopsie auch die Feinnadelaspiration eingesetzt. Wie hoch ist die Übereinstimmung zwischen den beiden Verfahren?
a. ca. $60 \%$
b. ca. $75 \%$
c. ca. $80 \%$
d. ca. $90 \%$
e. ca. $95 \%$

\section{Frage 8}

Die Prostatektomie beim Prostatakarzinom führt in der Regel zu welcher Komplikation?
a. Strangurie
b. Hämaturie
c. Inkontinenz
d. Pollakisurie
e. Nykturie

\section{Frage 9}

Welche Art der Neoplasie kommt am häufigsten in der Prostata beim Rüden vor?
a. Übergangsepithelkarzinom
b. Plattenepithelkarzinom
c. undifferenziertes Sarkom
d. Adenokarzinom
e. Hämangiosarkom

\section{Frage 10}

Das durchschnittliche Alter der Rüden, die an einem Prostatakarzinom erkrankt sind, liegt zum Zeitpunkt der Diagnosestellung bei welchem Alter?
a. 6 Jahre
b. 7 Jahre
c. 8 Jahre
d. 9 Jahre
e. 10 Jahre 


\section{Das Prostatakarzinom des Hundes}

Franziska Eberhardt, Ingmar Kiefer

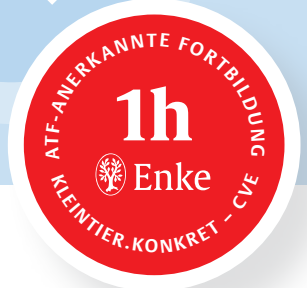

A Lernerfolgskontrolle

\section{B Teilnehmer}

\section{Bitte kreuzen Sie die richtigen Antworten an! Es ist jeweils nur 1 Antwort pro Frage richtig!}

\begin{abstract}
$\begin{array}{lllllllll}\text { Frage } & 1 & \text { a } & \text { b } & \text { c } & \text { d } & \text { e }\end{array}$
Frage 2 a b c d e

Frage 3 a b c d e

$\begin{array}{lllllll}\text { Frage } & 4 & \text { a } & \text { b } & \text { c } & \text { d } & \text { e }\end{array}$

$\begin{array}{lllllll}\text { Frage } & 5 & \text { a } & b & c & d & e\end{array}$
\end{abstract}

Titel | Name | Vorname

Straße | Hausnummer

PLZ | Ort

Beruf

\section{Ihr Ergebnis wird vom Verlag ausgefültt}

Sie haben von $\square \quad$ Fragen bestanden und 1 ATF-Stunde erhalten.

richtig beantwortet und somit

Stuttgart, den

\section{Teilnahmebedingungen für Abonnenten der kleintier konkret kostenlos}

Für diese Fortbildungseinheit können Sie 1 ATFFortbildungsstunde anerkannt bekommen. Hierfür

- müssen mindestens $70 \%$ der Fragen richtig beantwortet sein.

- muss der Antwortbogen vollständig ausgefüllt sein. Unvollständig ausgefüllte Bögen können nicht berücksichtigt werden!

\section{E Erklärung}

Ich versichere, dass ich die Beantwortung der Fragen selbst und ohne Hilfe durchgeführt habe.

\section{- muss im markierten Feld* Ihre Abonnen- tennummer eingetragen oder eine kleintier konkret-CVE-Wertmarke aufgeklebt sein.}

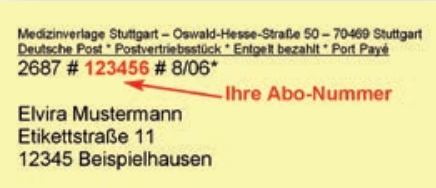

$\begin{array}{llllllll}\text { Frage } & 6 & \text { a } & \text { b } & c & d & \text { e }\end{array}$

$\begin{array}{llllllll}\text { Frage } & \mathbf{a} & \mathrm{b} & \mathrm{c} & \mathrm{d} & \mathrm{e}\end{array}$ $\begin{array}{lllllllll}\text { Frage } & 8 & \text { a } & \text { b } & c & d & e\end{array}$ $\begin{array}{llllllll}\text { Frage } & 9 & \text { a } & \text { b } & \text { c } & \text { d } & \text { e }\end{array}$ Frage 10 a kleintier konkret-CVE-Wertmarken für NichtAbonnenten können beim Verlag zu folgenden Bedingungen erworben werden: 6erPackWertmarken, Preis 49,95€ inkl. MWSt., Artikel-Nr. 903000.

Bitte richten Sie die Bestellungen an: MVS Medizinverlage Stuttgart,

KundenServiceCenter Buch, Postfach 3011 20, 70451 Stuttgart.

* Nicht-Abonnenten bitte hier kleintier konkret-CVE-Wertmarke aufkleben, Abonnenten bitte Abonnentennummer eintragen 